Published online 2015 April 22.

Case Report

\title{
Imaging Findings of an Unusually Located Hydatid Cyst Presented as a Sacrococcygeal Mass
}

\author{
Guliz Yilmaz ${ }^{1, *}$; Suha Halil Akpinar ${ }^{1}$ \\ ${ }^{1}$ Department of Radiology, Faculty of Medicine, Near East University, Nicosia, Turkey \\ ${ }^{*}$ Corresponding author: Guliz Yilmaz, Department of Radiology, Faculty of Medicine, Near East University, Nicosia, Turkey. Tel: +90-3926751000, Fax: +90-3926751090, E-mail: \\ glz.yilmaz@hotmail.com
}

Received: May 14, 2014; Revised: July 20, 2014; Accepted: September 14, 2014

\begin{abstract}
Hydatid disease (HD) is a parasitic infection that is most commonly caused by the larval stage of Echinococcus granulosus. Unusual location for this disease can cause diagnostic and therapeutic problems. We herein report a case of sacrococcygeal HD at an unusual location in a 30-year-old woman. She was evaluated using computed tomography (CT) imaging and magnetic resonance imaging (MRI) after the demonstration of the lucent bone lesion on plain pelvic radiography. There was an expansile lytic mass without contrast enhancement suggesting a cystic mass in the sacrococcygeal region. Medical history revealed that she had undergone surgery for liver HD and the serological test results were diagnostic for HD. In light of this, no surgery was carried out for this cystic mass and she was followed with the diagnosis of sacrococcygeal HD.
\end{abstract}

Keywords: Echinococcosis; Computed Tomography; X-Ray; Magnetic Resonance Imaging

\section{Introduction}

Hydatid disease (HD) is an endemic parasitic infection that is most commonly caused by the larval stage of Echinococcus granulosus $(1,2)$. Cysts are mostly found in the liver (55-60\%) and lungs (30\%) and involvement of the bones is very rare $(1-4 \%)(3,4)$. The unusual site of HD can cause misdiagnoses and complications (4). We herein present this case as a reminder that hydatid cyst that is located in the sacrococcygeal region can be confused with chordoma whose most common location in the spine is the sacrococcygeal area.

\section{Case Presentation}

A 30-year-old woman with a high socio-cultural status presented with the complaints of progressive left lower limb pain. The radiating intermittent pain started one year ago and progressively increased in intensity. On admission, her neurological examination revealed hypoesthesia and radicular pain of the left leg on admission. The pelvic computed tomography (CT) was carried out to detect the sacral mass after the lucent bone lesion was spotted on plain pelvic radiography. There was an expansile lytic mass with cortical bone destruction in the sacrococcygeal region (Figure 1). An abdominopelvic magnetic resonance imaging (MRI) was performed, which showed a lobulated and septated cystic mass at the sacrococcygeal region, hyperintense on T2 weighted (W) and hypointense on T1W sequences.

There was no contrast enhancement and the cystic mass was expanding the neural foramina between the S4 ver- tebra and coccyx (Figure 2A-D). The displacement and the compression of the lumbosacral nerve roots was revealed by MRI. There were no additional pathological findings on the abdominal MRI. She also had a history of liver HD operation 6 years ago and the positive serological test results obtained at the time of pelvic mass imaging were diagnostic for HD. The cystic mass was diagnosed as HD based on the imaging findings and serological results. The patient received albendazole (10 $\mathrm{mg} / \mathrm{kg} /$ day) for 6 months with close observation of the liver enzymes. There was no change in size at the one year follow-up period and the patient rejected the operation.

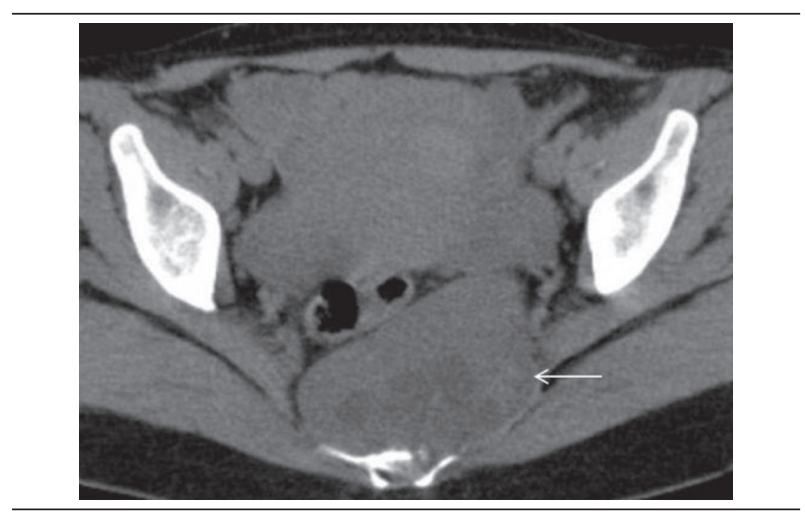

Figure 1. A 30-year-old woman with progressive radiating left lower limb pain and hypoesthesia and a lucent sacral bone lesion on plain pelvic radiography. Axial CT scan of the pelvis shows an expansile lytic heterogeneous mass with cortical bone destruction in the sacrococcygeal region (arrow).

Copyright (C) 2015, Tehran University of Medical Sciences and Iranian Society of Radiology. This is an open-access article distributed under the terms of the Creative Commons Attribution-NonCommercial 4.0 International License (http://creativecommons.org/licenses/by-nc/4.0/) which permits copy and redistribute the material just in noncommercial usages, provided the original work is properly cited. 

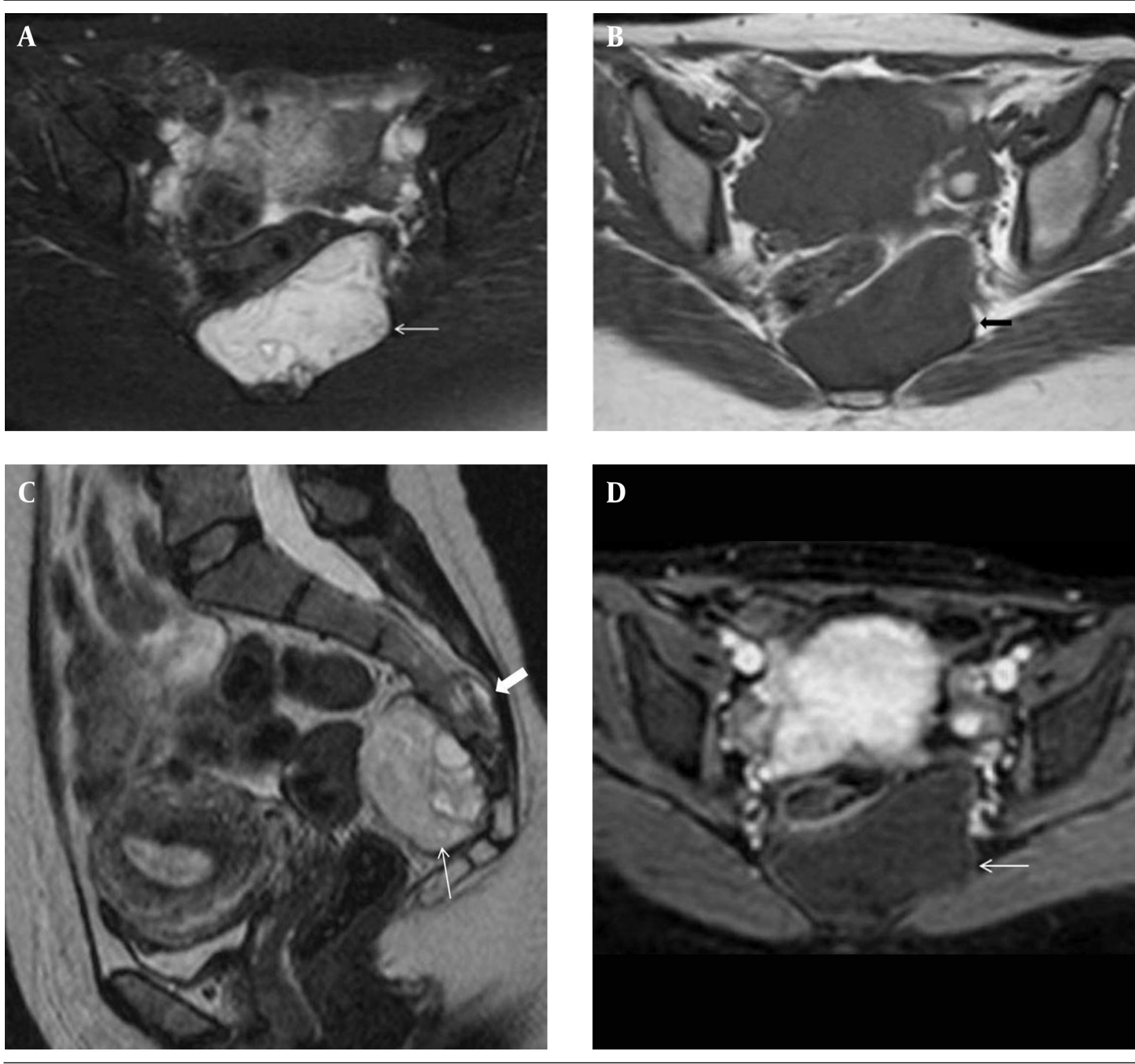

Figure 2. Axial pelvic MRI demonstrates a lobulated and septated cystic mass at the sacrococcygeal region. A, hyperintense on T2W fat saturated sequence. B, hypointense on T1W sequence (arrow). C, Sagittal T2W pelvic MRI shows a hyperintense multicystic mass (thin arrow) and cystic component at the sacrococcygeal vertebra (thick arrow). D, Axial contrast-enhanced T1W MRI reveals that there was no contrast enhancement suggesting cystic mass (arrow).

\section{Discussion}

HD is a parasitic infection that is most commonly caused by the larval stage of Echinococcus granulosus and less frequently Echinococcus alveolaris. It is still an important public health problem and endemic illness in countries such as China, Central Asia, the Middle East, the Mediterranean region, Eastern Africa and parts of South America $(1,2)$. Echinococcal eggs of the adult worm are present in the small intestine of definitive hosts. The definitive hosts are canines that excrete the eggs with the feces. When these eggs are ingested by intermediate hosts like sheep, cattle, goat or humans, they penetrate the intestinal mucosa of these hosts and enter the blood and lymphatic circulation. They are carried to the various organs, most commonly the liver and lungs where they develop into forms of hydatid cysts (2, $5)$. Hydatid cysts are found in the liver (55-60\%), lungs (30\%), kidneys (2.5-3\%), bones (1-4\%), and in other organs such as the brain $(1-2 \%)(3,4)$. HD is detected in the spine in nearly $1 \%$ of all cases. The distribution of the disease in the spine is $10 \%$ in the cervical area, $50 \%$ in the thoracic area, $20 \%$ in the lumbar area and $20 \%$ in the sacrum (6). Spinal HDs are classified into five groups as intramedullary hydatid cysts, intradural extramedullary hydatid cysts, extradural intraspinal hydatid cysts, hydatid cysts 
of the vertebrae and paravertebral hydatid disease (7). Our case showed involvement of the vertebral, paravertebral and intraspinal regions. Chordoma is the most common tumor of the sacrococcygeal region and may mimic cysts on CT or MRI (8). Wherever cysts are found in the body especially in the endemic countries, HD should be considered as the leading cause. Our case was an example of a very unusual location of HD as a sacrococcygeal cystic mass. Lumbar disc herniation is the most common reason for sciatica, lower back pain and neurological symptoms (9). If there is no disc pathology, the vertebral column and pelvis should be examined to define the reason. Although very rare, in patients presenting with the radiating limb pain and neurological symptoms that are caused by the compression of spinal cord or nerve roots, HD is to be considered in the differential diagnosis in endemic countries (6). The recurrence of HD occurs usually within 2 years after the previous HD operation (8-22\%), whereas our case was operated for liver HD 6 years ago (5). We diagnosed our case as HD with the imaging findings and furthermore the history of HD operation and serological test results. Recognition of HD, especially in endemic regions can be helpful for the diagnosis of cystic masses in the whole body. Preoperative diagnosis of HD is essential to prevent the complications of this disease.

\section{Authors' Contributions}

Guliz Yilmaz carried out patient assessment and data gathering. Guliz Yilmaz and Suha Halil Akpinar performed manuscript writing.

\section{References}

1. Lewall DB. Hydatid disease: biology, pathology, imaging and classification. Clin Radiol.1998;53(12):863-74.

2. Eckert J, Deplazes P. Biological, epidemiological, and clinical aspects of echinococcosis, a zoonosis of increasing concern. Clin Microbiol Rev. 2004;17(1):107-35.

3. Barrett NR, Thomas D. Pulmonary hydatid disease. Br J Surg. 1952;40(161):222-44.

4. Yuksel M, Demirpolat G, Sever A, Bakaris S, Bulbuloglu E, Elmas N. Hydatid disease involving some rare locations in the body: a pictorial essay. Korean J Radiol. 2007;8(6):531-40.

5. Terek MC, Ayan C, Ulukus M, Zekioglu O, Ozkinay E, Erhan Y. Primary pelvic hydatid cyst. Arch Gynecol Obstet. 2000;264(2):93-6.

6. Charles RW, Govender S, Naidoo KS. Echinococcal infection of the spine with neural involvement. Spine (Phila Pa 1976). 1988;13(1):47-9.

7. Iplikcioglu AC, Kokes F, Bayar A, Doganay S, Buharali Z. Spinal invasion of pulmonary hydatidosis: computed tomographic demonstration. Neurosurgery.1991;29(3):467-8.

8. Buyukgebiz O, Aktan AO, Dosluoglu H, Esemenli T, Soylemezoglu F. Sacrococcygeal chordoma. A case report and review of the literature. Marmara Med J.1991;2(4):75-7.

9. Hassan FO, Shannak A. Primary pelvic hydatid cyst: an unusual cause of sciatica and foot drop. Spine (Phila Pa 1976). 2001;26(2):230-2. 